\title{
LARGE-SCALE DYNAMO PRODUCED BY NEGATIVE MAGNETIC EDDY DIFFUSIVITIES
}

\author{
A. LANOTTE ${ }^{a, *}$, A. NOULLEZ ${ }^{a}$, M. VERGASSOLA ${ }^{a}$ \\ and A. WIRTH ${ }^{\mathrm{b}}$ \\ ${ }^{a}$ C.N.R.S., Observatoire de la Côte d'Azur, B.P. 4229, 06304 Nice Cedex 4, \\ France; ${ }^{b}$ Institut für Meereskunde, Kiel, Germany
}

The existence of incompressible flow producing negative magnetic eddy diffusivities is demonstrated. This provides for a dynamo mechanism, alternative to $\alpha$-type effects, requiring neither the presence of mean helicity nor the breaking of parity invariance. In the kinematic dynamo phase, the magnetic field grows exponentially with a growth rate proportional to the square of the wavenumber. The concrete example, analyzed by means of multiscale techniques, is a parity-invariant flow of the Taylor-Green type.

Keywords: Dynamo processes; stability; turbulent diffusion

\section{INTRODUCTION}

The generation of large-scale magnetic fields is one of the key issues in magnetahydrodynamics (e.g., Moffatt, 1978). The nature and the properties of self-inductive mechanisms capable of maintaining a persistent magnetization are of particular importance for cosmical objects, such as stars or galaxies (Parker, 1979; Zeldovich et al., 1983). For the investigation of magnetic fields growth, the Lorentz reaction of the magnetic field $\mathbf{B}$ on the velocity $\mathbf{v}$ can be neglected (at least initially) and the resulting dynamics is commonly known as the

\footnotetext{
${ }^{*}$ Corresponding author. e-mail: lanotte@obs-nice.fr
} 
kinematic dynamo problem. The velocity field $\mathbf{v}$ is indeed prescribed $a$ priori as a function (deterministic or random) of the space-time variables $\mathbf{x}$ and $t$. The issue is to determine the characteristics of the possible growth of the large-scale components of $\mathbf{B}(\mathbf{x}, t)$ (large-scale dynamo) in terms of $\mathbf{v}$ properties. The major effect of the incompressible flow $\mathbf{v}$ on the mean magnetic field $\overline{\mathbf{B}}$ is to generate a mean electromotive force (e.m.f.) $\mathcal{E}$, influencing the evolution of $\overline{\mathbf{B}}$ according to the induction equation

$$
\partial_{t} \overline{\mathbf{B}}=(\nabla \times \mathcal{E})+\eta \nabla^{2} \overline{\mathbf{B}} .
$$

In a mean-field framework (Moffatt, 1978), the e.m.f. is expanded in a gradient series as

$$
\mathcal{E}_{i}=\alpha_{i j} \bar{B}_{j}+\beta_{i j l} \frac{\partial \bar{B}_{j}}{\partial x_{l}}+\cdots
$$

where the coefficients $\alpha_{i j}$ and $\beta_{i j l}$ are functionals of the velocity field $\mathbf{v}$. Higher-order terms involve more than one spatial gradient of $\overline{\mathbf{B}}$. The gradient expansion has a fully systematic status in scale-separated situations, i.e., when the velocity $\mathbf{v}$ has finite typical space-time scales and the scales of interest for $\overline{\mathbf{B}}$ are much larger than all of them. The first term in the expansion (2) is responsible for the well-known $\alpha$-effect (Steenbeck et al., 1966). If the $\alpha$ tensor does not identically vanish, the term associated to it in (1) (first-order in space variables) will indeed become dominant with respect to the second-order molecular diffusivity term at sufficiently large scales. In three dimensions, this leads to an exponential growth of $\overline{\mathbf{B}}$ with a growth rate proportional to the wavenumber $k$. Lack of parity-invariance is the central ingredient for $\alpha$-type instabilities. By parity-invariant flow we mean those fields $\mathbf{v}$ admitting a center of symmetry relative to which the simultaneous reversal $\mathbf{x} \mapsto-\mathbf{x}$ and $\mathbf{v} \mapsto-\mathbf{v}$ leaves the flow invariant (deterministically or statistically, depending on the case considered). When no such center can be found, parity invariance is broken. Since the e.m.f. $\mathcal{E}$ is a vector and $\overline{\mathbf{B}}$ is a pseudo-vector, such breaking is a necessary condition for $\alpha_{i j}$ not to vanish identically. By the same argument, the average value of the magnetic helicity $\mathbf{A}(\mathbf{x}, t) \cdot \mathbf{B}(\mathbf{x}, t)$ is guaranteed to vanish in parityinvariant situations. ${ }^{1}$ When the flow does not admit any center of

\footnotetext{
${ }^{1}$ In the relation above, $\mathbf{A}$ is the vector potential satisfying $\mathbf{B}=\boldsymbol{\nabla} \times \mathbf{A}$.
} 
symmetry, the mean value of (kinetic) helicity can vanish or not. The latter case generally leads to stronger dynamos at large scales, with growth rates proportional to the wavenumber $k$ (and not $k^{2}$ as in the former case). It is therefore commonly accepted that helicity plays an important role for $\alpha$-type instabilities (Gilbert et al., 1988). Note also that $\alpha$ instabilities have a vanishing threshold in the magnetic Reynolds number, as the molecular diffusivity is subdominant at sufficiently large scales.

For parity-invariant flow, a possible large-scale dynamo cannot take place via the $\alpha$-effect and second-order terms in (2) become relevant. Inserting them into (1), a formally diffusive equation is obtained, where "formally" is meant to stress that the second-order operator on the right-hand side is not guaranteed to be (semi) negative-definite. It is certainly negative definite when the molecular diffusivity is large enough to dominate turbulent effects. But when the magnetic Reynolds number is increased, it is conceivable that the turbulent term associated to the $\beta$ tensor could destabilize the large scales and quantitatively overwhelm the stabilizing molecular contribution, thus leading to dynamo, i.e., the growth of $\overline{\mathbf{B}}$. This circumstance would correspond to a negative magnetic eddy diffusivity and would provide for a dynamo mechanism not requiring any breaking of parity invariance and/or mean helicity. The purpose of the present paper is to address the question formulated in Section 7.4 of (Moffatt, 1978) whether this dynamo mechanism is physically realizable.

The answer to the equivalent question for other physical situations is known. For the transport of passive scalars, the effect of an incompressible velocity $\mathbf{v}$ is always stabilizing, i.e., the eddy diffusivity is larger than the molecular diffusivity (Mc Laughlin et al., 1985). For time-independent potential flow, the eddy diffusivity is smaller than the molecular diffusivity, but it cannot become negative (Vergassola and Avellaneda, 1997). Another relevant situation is momentum transport in the Navier-Stokes equations. The effect analogous to the $\alpha$ effect is the so-called AKA effect (Frisch et al., 1987), which also disappears in the presence of parity-invariance. The existence of negative eddy viscosities is now well established, in two and in three dimensions, both for isotropic and anisotropic flow (Meshalkin and Sinai, 1961; Nepomnyashchy, 1976; Sivashinsky, 1985; Gama et al., 1994; Wirth et al., 1995). The existence of negative eddy viscosities 
points in favor of negative magnetic eddy diffusivities, but this indication should be taken with caution. Indeed, for simple parallel flows (depending on a single coordinate), the eddy viscosity is for example known to be negative, but the origin of the instability (at least in this case) comes entirely from the non-local pressure term (e.g., Meshalkin and Sinai, 1961; Dubrulle and Frisch, 1991), which is absent in the kinematic dynamo problem. This important difference is confirmed in the Appendix, where the same problem of parallel flow is solved for magnetic fields and no dynamo is indeed present.

Arguments in favor of negative magnetic eddy diffusivities were previously given in Roberts (1972) and Kraichnan (1976). The attention in Roberts (1972) was mainly concentrated on $\alpha$-type dynamo, but some evidence was also given that magnetic fields could grow when orders higher than the first in (2) are relevant. A three-scale argument was considered in Kraichnan (1976) to show that fluctuations of the $\alpha$-coefficient at intermediate scales could give a negative contribution to the eddy diffusivity on large scales, but no definite conclusion could be drawn. Here, we shall analyze the problem using multiscale techniques, presented in Section 2. The advantage is that the nature and the order of the large-scale dynamics can be systematically identified and the calculation of the eddy-diffusivity tensor is reduced to the solution of auxiliary equations on the elementary periodicity cell. In Section 3, numerical simulations of the auxiliary equations are used to investigate a steady variant of the Taylor-Green vortex, which is found to produce a negative magnetic eddy diffusivity above a critical Reynolds number.

\section{MULTISCALE FORMALISM}

The aim of this section is to present the multiscale formalism that will be later exploited for the calculation of magnetic eddy diffusivities. The incompressible velocity field $\mathbf{v}(\mathbf{x}, t)$ in the kinematic dynamo equation

$$
\partial_{t} \mathbf{B}=\boldsymbol{\partial} \times(v \times \mathbf{B})+\eta \partial^{2} \mathbf{B}, \quad \boldsymbol{\partial} \cdot \mathbf{B}=0,
$$

is supposed to have a finite typical length scale $\ell_{0}, e . g$., to be $\ell_{0}$ periodic, and we shall be interested in the dynamics of the magnetic 
field $\mathrm{B}$ at scales $L \gg \ell_{0}$. The ratio $\ell_{0} / L \equiv \varepsilon \ll 1$ provides a small parameter that can be exploited for a perturbative solution. The perturbation being singular (e.g., Bender and Orszag, 1978), it is necessary to treat the problem by singular perturbation methods (see Nayfeh, 1973; Van Dyke, 1975). The most convenient method for the case at stake is the multiscale technique, also known as homogenization (Bensoussan et al., 1978). In addition to the original variables $(\mathbf{x}, t)$ ("fast" variables) characterizing the basic flow $\mathrm{v}$, a new set of space-time variables $\mathbf{X}, T$ ("slow" variables) is introduced. The rationale in their choice is that the large-scale dynamics should be $\mathrm{O}(1)$ in the new variables, e.g., $\mathbf{X}=\varepsilon \mathbf{x}$. The prescription that resolves the singularity is to treat the two sets of variables, fast and slow, as independent throughout the perturbative expansion. The enlargement of degrees of freedom is compensated by the presence of non-trivial solvability conditions in the resulting equations, giving the large-scale evolution equations.

Let us implement the previous procedure for the kinematic dynamo equation (3). Since we are interested in eddy diffusivities we shall impose the velocity field $\mathbf{v}$ to be parity-invariant. This ensures the $a b-$ sence of the $\alpha$ effect and makes the dynamics at large scales expected to be second-order in the space variables. The appropriate rescalings for the slow variables ${ }^{2}$ are then

$$
\mathbf{X}=\varepsilon \mathbf{x} ; \quad T=\varepsilon^{2} t
$$

Treating fast and slow variables as independent implies the following chain rule for the derivatives

$$
\boldsymbol{\partial} \rightarrow \boldsymbol{\partial}+\varepsilon \nabla ; \quad \partial_{t} \rightarrow \partial_{t}+\varepsilon^{2} \partial_{T}
$$

Here, we denote by the symbol $\partial$ the derivatives with respect to fast variables and by $\left(\nabla, \partial_{T}\right)$ those with respect to slow variables. The solution is then sought in the form of a perturbative series in $\varepsilon$ :

$$
\mathbf{B}=\mathbf{B}^{(0)}+\varepsilon \mathbf{B}^{(1)}+\varepsilon^{2} \mathbf{B}^{(2)}+\cdots,
$$

\footnotetext{
${ }^{2}$ In the presence of the $\alpha$ effect, two slow times should be introduced: $T_{1}=\varepsilon t$, to capture the $\alpha$ dynamics and $T_{2}=\varepsilon^{2} t$ for the eddy diffusivity contributions.
} 
where the $\mathbf{B}^{(n)}$ functions depend on both fast and slow variables. Inserting the expansion (6) and the rule for derivatives (5) into the original equation (3), we derive the following hierarchy of equations:

$$
\begin{aligned}
\mathrm{O}\left(\varepsilon^{0}\right) \quad \mathcal{A} \tilde{\mathbf{B}}^{(0)} & =\left\langle\boldsymbol{B}^{(0)}\right\rangle \cdot \partial \mathbf{v} \\
\mathrm{O}\left(\varepsilon^{1}\right) \quad \mathcal{A} \mathbf{B}^{(1)}= & -\mathbf{v} \cdot \nabla \mathbf{B}^{(0)}+2 \eta \boldsymbol{\partial} \cdot \nabla \tilde{\mathbf{B}}^{(0)}+\left(\boldsymbol{\nabla} \cdot \tilde{\mathbf{B}}^{(0)}\right) \mathbf{v} \\
\mathrm{O}\left(\varepsilon^{2}\right) \quad \mathcal{A} \mathbf{B}^{(2)}= & -\mathbf{v} \cdot \nabla \mathbf{B}^{(1)}+2 \eta \boldsymbol{\partial} \cdot \nabla \mathbf{B}^{(1)}+\left(\boldsymbol{\nabla} \cdot \mathbf{B}^{(1)}\right) \mathbf{v} \\
& -\partial_{\mathrm{T}} \mathbf{B}^{(0)}+\eta \nabla^{2} \mathbf{B}^{(0)} .
\end{aligned}
$$

The operator $\mathcal{A}$ is simply the induction operator acting on fast variables:

$$
\mathcal{A} \bullet=\partial_{\imath} \bullet-\boldsymbol{\partial} \times(\mathbf{v} \times \bullet)-\eta \partial^{2} \bullet,
$$

and $\mathbf{B}^{(0)}=\left\langle\mathbf{B}^{(0)}\right\rangle+\tilde{\mathbf{B}}^{(0)}$ has been decomposed in its mean and fluctuating parts.

For the divergence-free conditions, we obtain at the various orders of $\varepsilon$

$$
\begin{array}{ll}
\mathrm{O}\left(\varepsilon^{0}\right) & \boldsymbol{\partial} \cdot \mathbf{B}^{(0)}=0, \\
\mathrm{O}\left(\varepsilon^{1}\right) & \boldsymbol{\partial} \cdot \mathbf{B}^{(1)}+\boldsymbol{\nabla} \cdot \mathbf{B}^{(0)}=0, \\
\mathrm{O}\left(\varepsilon^{2}\right) & \boldsymbol{\partial} \cdot \mathbf{B}^{(2)}+\nabla \cdot \mathbf{B}^{(1)}=0 .
\end{array}
$$

Note that these conditions mix different orders of $\mathbf{B}^{(n)}$, implying, for example, that $\partial \times\left(\mathbf{v} \times \mathbf{B}^{(1)}\right) \neq \mathbf{v} \cdot \partial \mathbf{B}^{(1)}-\mathbf{B}^{(1)} \cdot \partial \mathbf{v}$.

The operator $\mathcal{A}$ having derivatives at the left of all terms, the average $\langle\mathcal{A} \mathbf{f}\rangle$ vanishes for any $\mathbf{f}$ having the same periodicities as $\mathbf{v}$. A necessary solvability condition for the auxiliary equations of the hierarchy is thus

$$
\mathcal{A} \mathbf{f}=\mathbf{g} \stackrel{\text { solvability }}{\longrightarrow}\langle\mathbf{g}\rangle=\mathbf{0}
$$


where $\langle\bullet\rangle$ denotes the average over the periodicities of the basic flow $\mathbf{v}$. In more general terms, by the Fredholm alternative theorem, the righthand side $g$ should be orthogonal to constant functions, that constitute the null-space of the operator $\mathcal{A}^{\dagger}$, adjoint of $\mathcal{A}$.

The first solvability condition at $\mathrm{O}\left(\varepsilon^{0}\right)$ is automatically satisfied since $\langle\hat{\delta} \mathbf{v}\rangle=0$. Using the linearity of the equation, its solution can be generally written in the form

$$
\tilde{B}_{i}^{(0)}=S_{i j}(\mathbf{x}, t)\left\langle B_{j}^{(0)}\right\rangle,
$$

with the field $S_{i j}$ having zero mean and satisfying the equation

$$
\mathcal{A} S_{i j}(\mathbf{x}, t)=\partial_{j} v_{i}
$$

At the next order $\mathrm{O}\left(\varepsilon^{1}\right)$, the solvability condition

$$
\boldsymbol{\nabla} \times\left\langle\mathbf{v} \times \tilde{\mathbf{B}}^{(0)}\right\rangle=\mathbf{0}
$$

or equivalently using (15)

$$
\left\langle v_{i}(\mathbf{x}) S_{j l}(\mathbf{x})\right\rangle-\left\langle v_{j}(\mathbf{x}) S_{i l}(\mathbf{x})\right\rangle=0,
$$

would in general be non trivial, corresponding to the $\alpha$ effect. However, if the velocity is parity invariant $\mathbf{v}(-\mathbf{x})=-\mathbf{v}(\mathbf{x})$, from (16) it follows that $S_{i j}(-\mathbf{x})=S_{i j}(\mathbf{x})$ and the average in (18) therefore vanishes. Using again the linearity of the equation, we can write its solution in the form

$$
B_{i}^{(1)}=\Gamma_{i j l}(\mathbf{x}, t)\left(\nabla_{j}\left\langle B_{l}^{(0)}\right\rangle\right),
$$

where the field $\Gamma_{i j l}(\mathbf{x})$ is the zero-mean solution of

$$
\mathcal{A} \Gamma_{i j l}(\mathbf{x}, t)=-\delta_{i l} v_{j}-v_{j} S_{i l}+v_{i} S_{j l}+2 \eta \partial_{j} S_{i l} .
$$

Finally, from the solvability condition at $O\left(\varepsilon^{2}\right)$ we obtain the evolution equation for the large-scale magnetic field $\left\langle\mathbf{B}^{(0)}\right\rangle(\mathbf{X}, T)$ :

$$
\partial_{T}\left\langle\mathbf{B}^{(0)}\right\rangle=-\left\langle\mathbf{v} \cdot \boldsymbol{\nabla} \mathbf{B}^{(1)}\right\rangle+\left\langle\mathbf{v} \cdot \mathbf{B}^{(1)}\right\rangle+\eta \nabla^{2}\left\langle\mathbf{B}^{(0)}\right\rangle .
$$


Substituting in (21) the expression (19), we obtain

$$
\partial_{T}\left\langle B_{i}^{(0)}\right\rangle(\mathbf{X}, T)=\eta_{i j l m}^{E} \nabla_{j} \nabla_{l}\left\langle B_{m}^{(0)}\right\rangle(\mathbf{X}, T)
$$

with the eddy diffusivity expressed in terms of averages of the $\Gamma$ field:

$$
\eta_{i j l m}^{E}=\eta \delta_{i m} \delta_{j l}-\left\langle v_{l} \Gamma_{i j m}\right\rangle+\left\langle v_{i} \Gamma_{l j m}\right\rangle .
$$

The large-scale dynamics is formally diffusive, as expected. Note that, according to the definition (23), the eddy diffusivity $\eta^{E}$ is the sum of the molecular contribution $\eta$ and those due to the presence of the small-scale velocity field $\mathbf{v}$. Since the transported quantity is a vector, the eddy diffusivity is a fourth-order tensor, whose entries are averages of functions depending only on fast variables. For a generic velocity field, the tensor $\eta_{i j / m}^{\mathcal{E}}$ will not be isotropic, i.e., the eddy diffusivity will depend on the direction. To single out this dependence, it is useful to decompose a generic field $\mathbf{B}$ on the Fourier basis and note that we can look for eigenfunctions of (22) in the form of plane waves:

$$
\left\langle\mathbf{B}^{(0)}(\mathbf{X}, T)\right\rangle=\mathbf{b} \exp \left[\mathbf{i k} \cdot \mathbf{X}-\eta^{E}(\hat{\mathbf{k}}) k^{2} T\right] .
$$

Plugging (24) into (22), for each unit vector $\hat{\boldsymbol{k}}$ we obtain the following eigenvalue problem:

$$
\begin{gathered}
M_{i m}(\hat{\mathbf{k}}) b_{m}=\eta^{E}(\hat{\mathbf{k}}) b_{i}, \\
M_{i m}(\hat{\mathbf{k}})=\eta_{i j l m}^{E} \hat{k}_{j} \hat{k}_{l} .
\end{gathered}
$$

The non-trivial part of this $(d \times d)$ matrix lives in fact on the $(d-1)$ dimensional sub-space perpendicular to the vector $\mathbf{k}$, the latter having zero eigenvalue. In three dimensions, once the entries of the $\eta^{E}$ tensor are known, the calculation of the eddy diffusivity in a given direction reduces therefore to the simple diagonalization of a $2 \times 2$ matrix.

\section{TAYLOR-GREEN FLOWS}

In this section we shall discuss the behavior of magnetic eddy diffusivities for the Taylor-Green flow, and a slight variant of it that will be shown to produce a negative magnetic eddy diffusivity. 
The Taylor-Green flow $\mathbf{v}^{\mathrm{TG}}$ :

$$
v_{x}^{\mathrm{TG}}=\sin x \cos y \cos z, \quad v_{y}^{\mathrm{TG}}=-\cos x \sin y \cos z, \quad v_{z}^{\mathrm{TG}}=0,
$$

was thoroughly investigated in the study of vorticity enhancement by vortex-line stretching and the consequent production of small-scale eddies (Brachet $e$ t al., 1983). The planes $x, y$ or $z=n \pi$ (with $n$ integer) are of mirror symmetry and they constitute the faces of the so-called impermeable box since no fluid crosses these boundaries. The overall geometry of the flow resembles that of a now classical experimental configuration consisting of a shear layer between two counter-rotating disks. The many symmetries of the Taylor-Green flow (see Brachet $e t$ al., 1983) ensure that when $\mathbf{v}^{\mathrm{TG}}$ is used as initial condition in a Navier-Stokes simulation, the resulting flow admits the following Fourier expansion (when bifurcations breaking these symmetries are neglected):

$$
\begin{aligned}
& v_{x}=\sum_{m=0}^{\infty} \sum_{n=0}^{\infty} \sum_{p=0}^{\infty} u_{x}(m, n, p) \sin m x \cos n y \cos p z \\
& v_{y}=\sum_{m=0}^{\infty} \sum_{n=0}^{\infty} \sum_{p=0}^{\infty} u_{y}(m, n, p) \cos m x \sin n y \cos p z \\
& v_{z}=\sum_{m=0}^{\infty} \sum_{n=0}^{\infty} \sum_{p=0}^{\infty} u_{z}(m, n, p) \cos m x \cos n y \sin p z
\end{aligned}
$$

where the vector $\mathrm{u}$ vanishes unless the integers $(m, n, p)$ are all even or all odd. The full MHD nonlinear problem with a Taylor-Green forcing on the velocity was recently numerically investigated in for example Nore et al. (1997). For very high viscosities only the basic mode of the forcing is active, while more and more modes other than the basic one are excited in the expansion (28) when the Reynolds number is increased (the non-basic modes will be referred to, in the sequel, as recirculation modes). The important observation made in Nore et al. (1997) is that the magnetic field grows for sufficiently large Reynolds numbers when the forcing is not on the smallest wavenumber of the box. Furthermore, the fastest growing mode of the magnetic field has a component on the smallest wavenumber, suggesting a sort of scale 
separation. These were our motivations in considering the following generalization (with the two most energetic recirculation modes) of the Taylor-Green flow

$$
\begin{aligned}
v_{x}= & \sin x \cos y \cos z+A \sin 2 x \cos 2 z \\
& +B[\sin x \cos 3 y \cos z+(5 / 13) \sin 3 x \cos y \cos z] \\
v_{y}= & -\cos x \sin y \cos z+A \sin 2 y \cos 2 z \\
& -B[\cos 3 x \sin y \cos z+(5 / 13) \cos x \sin 3 y \cos z] \\
v_{z}= & -A \sin 2 z(\cos 2 x+\cos 2 y) \\
& +B[(2 / 13) \cos x \cos 3 y \sin z-(2 / 13) \cos 3 x \cos y \sin z],
\end{aligned}
$$

where $A$ and $B$ are free parameters. The other constants, multiples of $1 / 13$, could also have in principle been left free. For simplicity, we decided to set them to the value they take when the solution of the Navier-Stokes equations with a Taylor-Green forcing is expanded in powers of the Reynolds number. Note that both the original TaylorGreen flow (27) and its variant (29) are parity-invariant (with respect to the origin). The vanishing of the mean helicity $\langle h(\mathbf{x})\rangle=\langle\mathbf{v} \cdot \boldsymbol{\omega}\rangle$ and the absence of $\alpha$ effect are therefore guaranteed. An important qualitative difference between the two flows is that, for (27), $h(\mathbf{x})$ itself vanishes everywhere and not just its average value. This is not the case for the flow (29) where helicity fluctuations, although globally vanishing, are locally quite strong. The magnetic eddy diffusivities for the flows (27) and (29) are calculated using the formalism of Section 2 and numerically solving the resulting auxiliary equations (16) and (20). The numerical code is a standard pseudo-spectral one, with the classical 2/3 truncation procedure for dealiasing (Gottlieb and Orszag, 1977). Since magnetic Reynolds numbers (defined here as $R_{m}=1 / \eta$ ) turn out to be of order ten, only moderate resolutions $32^{3}$ and $64^{3}$ are needed. We checked by the energy spectra that truncation or finite size errors are negligible for the range of magnetic diffusivities considered; it was verified that the results for the two resolutions coincide within relative errors of few per cent.

To solve the auxiliary equations, the most convenient way is to apply repeatedly the evolution operator $\mathcal{A}$ with a sufficiently small timestep until the field settles down to a stationary state. Note that this requires all small-scale modes to be damped (which is certainly guaranteed if the Reynolds numbers is small enough). This very same 
assumption is indeed also implicitly assumed in the multiscale procedure itself: for the dynamo instability to be at large scales, and for multiscale techniques to be applicable, it is necessary for small scales to be stable. The validity of such an assumption can be checked $a$ posteriori.

Let us now turn to the discussion of the results. In Figure 1, to better highlight the effects of the small-scale velocity field on largescale transport, we have plotted the minimum, over the unit wavenumbers $\hat{\mathbf{k}}$, of the difference between the eddy diffusivity $\eta^{E}(\hat{\mathbf{k}})$, defined in (23), and the molecular diffusivity $\eta$. For both the Taylor-Green flow (27) and the flow (29) with only the first recirculating mode excited ( $A=1, B=0)$, such difference always remains positive, and $a$ fortiori so does the whole eddy diffusivity $\eta^{E}$. In particular, in the former case, the eddy diffusivity has a tendency to saturate to a finite value, while in the latter case, the tendency is to decrease. However, when the magnetic Reynolds number is increased above $R_{m} \simeq 11$, the

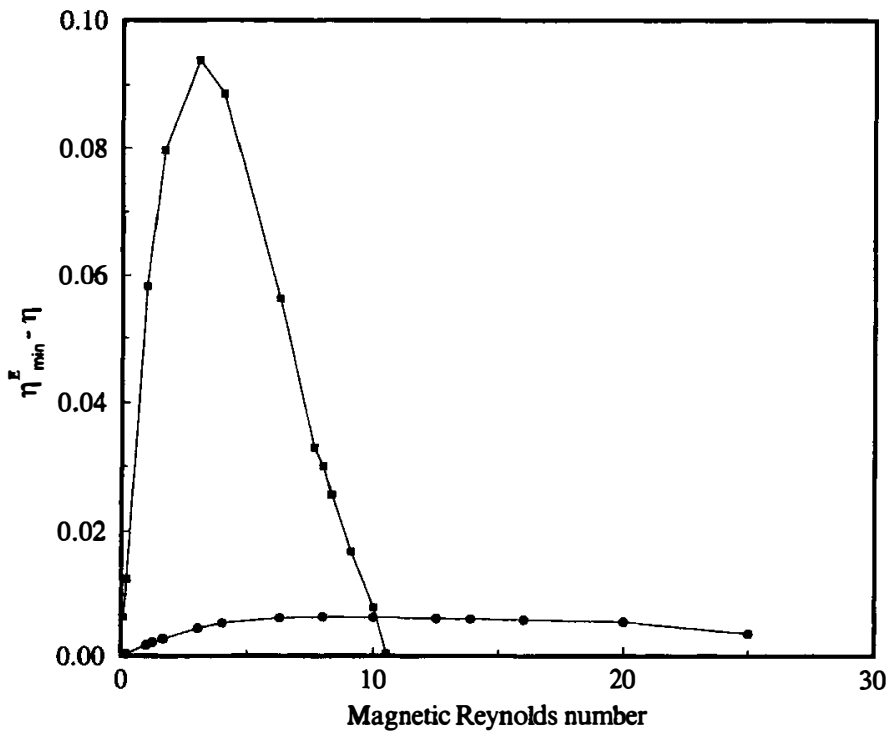

FIGURE 1 The difference $\left(\eta_{\min }^{E}-\eta\right)$ between the minimum (over the wavevector $\hat{\mathbf{k}}$ directions) of the eddy diffusivity $\eta^{E}(\hat{\mathbf{k}})$ and the molecular diffusivity $\eta v s$. the magnetic Reynolds number. According to the definition in (23), the difference $\left(\eta^{E}-\eta\right)$ gives the contribution to the eddy diffusivity arising from the presence of the small-scale velocity field $v$. Circles refer to the flow (27); squares to the flow (29) with $(A=1, B=0)$. 
kinematic dynamo equation becomes unstable at small scales, comparable to those of the velocity field, indicating that the primary dynamo mechanism is not acting at large scales. The evidence of negative magnetic eddy diffusivities is finally obtained for the flow (29) with $A=B=1$. As shown in Figure 2, for $R_{m} \geq R_{m}^{\text {crit }} \simeq 7.9$, there is at least one mode $\mathbf{k}$ having a negative eddy diffusivity $\eta^{E}(\hat{\mathbf{k}})$. As follows from (24), the corresponding eigencomponent of the magnetic field is exponentially amplified, with a growth-rate proportional to $k^{2}$. The modes lying in the $x y$ plane are the most unstable for any $R_{m}>R_{m}^{\text {crit }}$ (and, of course, they are the first to become unstable), with the corresponding eigencomponent of the magnetic field of the form $\mathbf{B}=$ $\left(0,0, B_{z} \exp i \mathbf{k} \cdot \mathbf{x}\right)$. For $R_{m}>R_{m}^{\text {crit }}$, there is in fact a whole basin of unstable directions $\hat{\mathbf{k}}$ around the most unstable one. At $R_{m}=10.52$, the angular amplitude of the basin is roughly $\pi / 6 \leq \theta \leq 5 \pi / 6$ (Fig. 3), where $\theta$ is the polar angle in spherical coordinates $(r, \theta, \phi)$.

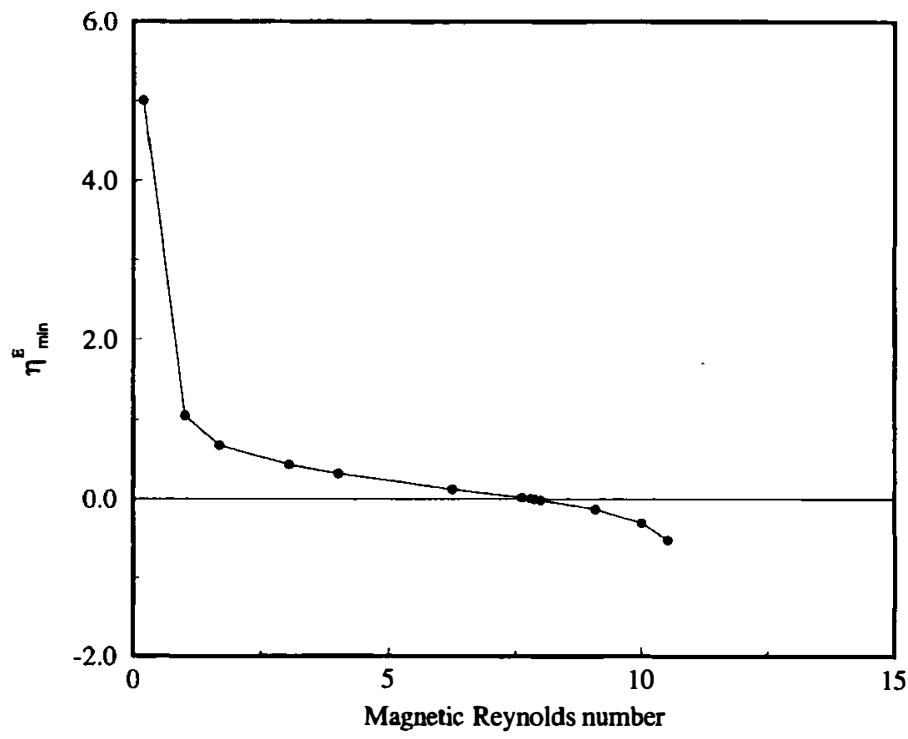

FIGURE 2 The minimum $\eta_{\text {min }}^{E}$ (over the wavevector $\hat{\mathbf{k}}$ directions) of the magnetic eddy diffusivity $\eta^{E}(\hat{\mathbf{k}}) v s$. the magnetic Reynolds number, for the flow (29) with $A=B=1$. As the magnetic eddy diffusivity $\eta^{E}$ defined in (23) already includes the molecular contribution, the change of sign of the curve at $R_{m}=R_{m}^{\text {crit }} \simeq 7.874$ corresponds to the threshold for the magnetic field growth. 


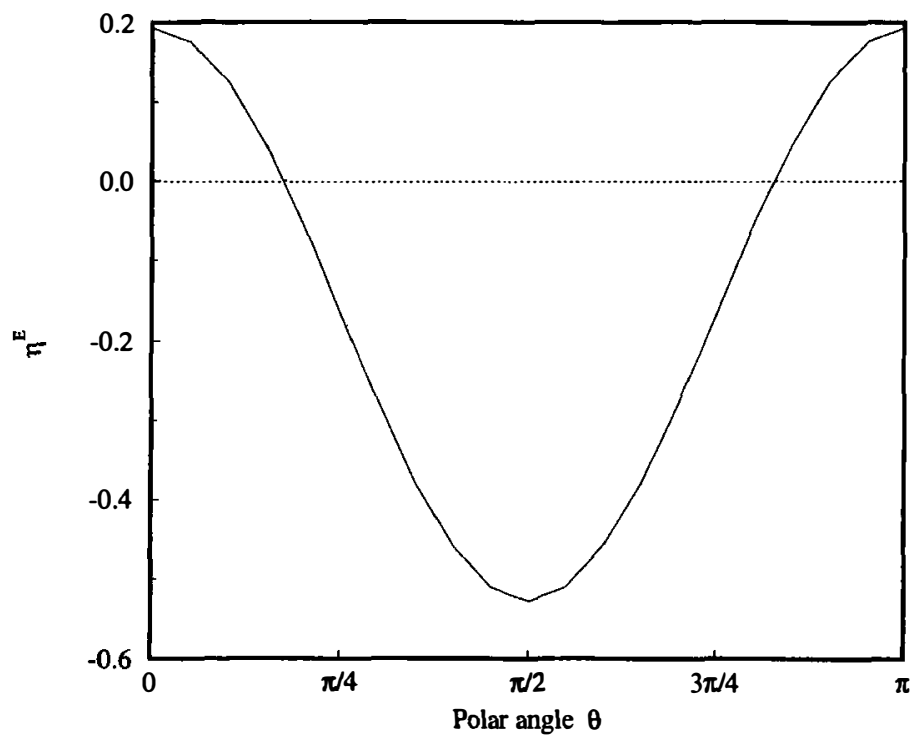

FIGURE 3 The variation of the eddy diffusivity $\eta^{E}(\hat{\mathbf{k}})$ with respect to the angle $\theta$ between the wavevector $\hat{\mathbf{k}}$ and the $\hat{z}$ axis, for $R_{m}=10.52$.

In conclusion, the possibility of a dynamo action by negative magnetic eddy diffusivities has been demonstrated. Helicity fluctuations, although globally vanishing, appear to play an important role in producing the negative eddy diffusivity, confirming the intuition of Kraichnan (1976). The negative eddy diffusivity mechanism does not require any breaking of parity invariance and offers therefore an alternative to $\alpha$-type effects.

\section{Acknowledgements}

We are most grateful to M. E. Brachet, U. Frisch, H. Politano and A. Pouquet for useful discussions. The work was supported by CNRS GdR "Mécanique des Fluides Géophysiques et Astrophysiques". Numerical simulations were performed at the Observatoire de la Côte d'Azur in the framework of the SIVAM project. AL was supported by the EEC under contract No. ERB-FMBI-CT96-0974. AW was supported by NASA grant NAG5-713. 


\section{References}

Bender, C. M. and Orszag, S. A., Advanced Mathematical Methods for Scientists and Engineers, Mc Graw-Hill (1978).

Bensoussan, A., Lions, J.-L. and Papanicolaou, G., Asymptotic Analysis for Periodic Structures, North-Holland, Amsterdam (1978).

Brachet, M. E., Meiron, D. I., Orszag, S. A., Nickel, B. G., Morf, R. H. and Frisch, U., "Small-scale structure of the Taylor-Green vortex," J. Fluid Mech. 130, 411-452 (1983).

Dubrulle, B. and Frisch, U., "Eddy viscosity of parity-invariant flow," Physical Rev. A 53, 5355 (1991).

Frisch, U., She, Z. S. and Sulem, P. L., "Large-scale flow driven by the anisotropic kinetic alpha effect," Physica D 28, 382- 392 (1987).

Gama, S., Vergassola, M. and Frisch, U., "Negative eddy viscosity in isotropically forced two-dimensional flow: linear and nonlinear dynamics," J. Fluid Mech. 180, 95-126 (1994).

Gilbert, A. D., Frisch, U. and Pouquet, A., "Helicity is unnecessary for alpha effect dynamos, but it helps," Geophys. Astrophys. Fluid Dynam. 42, 151-161 (1988).

Gottlieb, D. and Orszag, S. A., Numerical Analysis of Spectral Methods: Theory and Applications, Society for Industrial and Applied Mathematics, Philadelphia, Pennsylvania (1977).

Kazantzev, A. P., "Enhancement of magnetic fields by a conducting fluid," Sov. Phys. JETP 26, 1031 (1968).

Kraichnan, R. H., "Diffusion of weak magnetic fields by isotropic turbulence," J. Fluid Mech. 75, 657-676 (1976).

Mc Laughlin, D., Papanicolaou, G. C. and Pironneau, O., "Convection of microstructures and related problems," SIAM Journal of Appl. Math. 45, 780 (1985).

Meshalkin, L. D. and Sinai, Ya. G., "Investigation of the stability of a stationary solution of a system of equations for the plane movement of an incompressible viscous fluid," Appl. Math. Mech. 25, 1700-1705 (1961).

Moffatt, H. K., Magnetic field generation in electrically conducting fluids, Cambridge University Press (1978).

Nayfeh, A. H., Perturbation Methods, Wiley, New York (1973).

Nepomnyashchy, A. A., "On the stability of the secondary flow of a viscous fluid in an infinite domain," Appl. Math. Mech. 40, 886-891 (1976).

Nore, C., Brachet, M. E., Politano, H. and Pouquet, A., "Dynamo action in the TaylorGreen vortex near threshold," Phys. Plasmas 4(1), 1-3 (1997).

Parker, E. N., Cosmical Magnetic Fields, Oxford University Press (1979).

Roberts, G. O., "Dynamo action of fluid motions with two-dimensional periodicity," Phil. Trans. R. Soc. Lond. A271, $411-454$ (1972).

Sivashinsky, G. I., "Weak turbulence in periodic flows," Physica D 17, 243 (1985).

Steenbeck, M., Krause, F. and Rädler, K.-H., "A calculation of the mean electromotive force in an electrically conducting fluid in turbulent motion, under the influence of Coriolis forces," Z. Naturforsch. 21a, 369-376 (1966).

Van Dyke, M., Perturbation Methods in Fluid Mechanics, The Parabolic Press (1975).

Vergassola, M. and Avellaneda, M., "Scalar transport in compressible flow," Physica D 288, $249-264$ (1995).

Wirth, A., Gama, S. and Frisch, U., "Eddy viscosity of three-dimensional flow," $J$. Fluid Mech. 288, 249-264 (1995).

Zeldovich, Ya. B., Ruzmaikin, A. A. and Sokoloff, D. D., Magnetic Fields in Astrophysics, Gordon and Breach (1983). 


\section{APPENDIX A. SIMPLE ANALYTICALLY SOLVABLE CASES}

We shall briefly discuss here some simple cases where the calculation of the magnetic eddy diffusivity can be carried out analytically. For none of them does the eddy diffusivity turn out to be negative.

Let us start from the case when the magnetic Reynolds number $R_{m}$ is small enough to permit a perturbative expansion of the magnetic eddy diffusivity. The operator $\mathcal{A}$ in (10) then reduces to the heat operator $\mathcal{H} \equiv \partial_{t}-\eta \partial^{2}$ whose inversion on periodic functions having zero mean and a spectrum decaying sufficiently fast is well defined and easy to perform, e.g., in Fourier space. The solutions at the dominant order of the auxiliary equations (8) and (9) are thus:

$$
S_{i j}=\mathcal{H}^{-1}\left(\partial_{j} v_{i}\right) ; \quad \Gamma_{i j l}=\mathcal{H}^{-1}\left[-\delta_{i l} v_{j}+2 \eta \partial_{j} \mathcal{H}^{-1}\left(\partial_{l} v_{i}\right)\right]
$$

The expression of the eddy diffusivity tensor immediately follows from (23). For time-independent velocity fields, the turbulent contribution turns out to be positive definite. Indeed, the combination $I=-\eta_{i j l m}^{E} \hat{k}_{j} \hat{k}_{l} b_{i} b_{m}$ [which is the one involved in (26)] reduces to

$$
I=-\frac{1}{\eta} \hat{k}_{j} \hat{k}_{l} b_{i} b_{m}\left[\delta_{i m}\left\langle v_{l} \partial^{-2} v_{j}\right\rangle\right]=-\frac{1}{\eta} b^{2}\left\langle(\hat{\mathbf{k}} \cdot \mathbf{v}) \partial^{-2}(\hat{\mathbf{k}} \cdot \mathbf{v})\right\rangle,
$$

which is clearly positive.

For a generic flow, the previous formulae are just the first term of the expansion in the magnetic Reynolds number. There is however the special class of parallel flow where they hold in general. These flows are defined as:

$$
\mathbf{v}=\left[0, v_{2}(x), v_{3}(x)\right],
$$

where the simplifying feature is that they depend on a single coordinate (here arbitrarily chosen to be $x$ ). It follows that, in solving (8) and (9), the term $\partial \times(v \times \bullet)$ in $\mathcal{A}$ identically vanishes and the solution of the equations reduces again to inverting the heat operator. The previous perturbative formulae can therefore be simply carried over and the eddy diffusivity tensor is guaranteed to be positive definite by the general result (A.2). 
Let us finally recall that in the case when the velocity field is random and has a short-correlation time, an exact equation for the mean magnetic field exists (Katsantzev, 1968). The stretching term B· $\mathbf{\partial v}$ does not give any contribution and the resulting magnetic eddy diffusivity tensor is simply $\eta_{i j l m}^{E}=\eta \delta_{i m} \delta_{j l}+\delta_{i m} \int_{0}^{\infty}\left\langle v_{j}(\mathbf{x}, t) v_{l}(\mathbf{x}, 0)\right\rangle d t$, which is positive-definite (and position-independent for a homogeneous flow). 